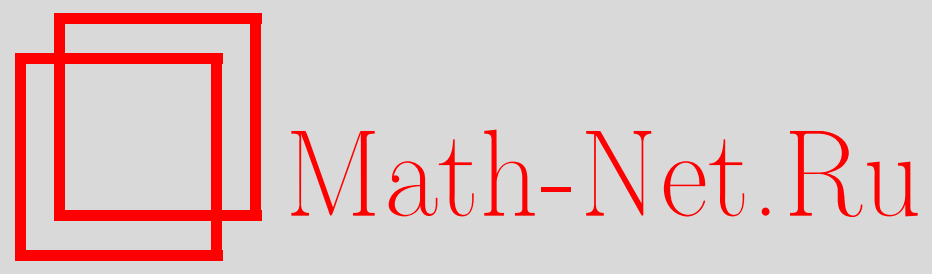

И. А. Круглов, Достижимость на конечном шаге предельных распределений для произведений случайных величин со значениями в конечной группе, Дискрет. матем., 2006, том 18, выпуск 3, 35-42

DOI: https://doi.org/10.4213/dm57

Использование Общероссийского математического портала Math-Net.Ru подразумевает, что вы прочитали и согласны с пользовательским соглашением http://www.mathnet.ru/rus/agreement

Параметры загрузки:

IP : 52.87 .193 .239

26 апреля 2023 г., 16:03:01

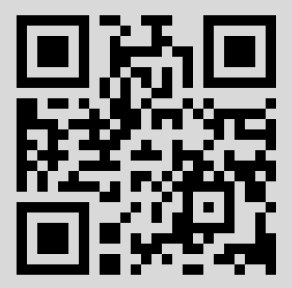




\title{
Достижимость на конечном шаге предельных распределений для произведений случайных величин со значениями в конечной группе
}

\author{
() 2006 г. И. А. Круглов
}

\begin{abstract}
В работе приведены необходимые и достаточные условия равновероятности на конечном шаге предельных распределений произведений случайных величин с значениями в конечной группе, распределения которых определяются цепью Маркова, при этом равномерное предельное распределение сосредоточено на соответствующих смежных классах исходной конечной группы по ее некоторым сопряженным подгруппам.

Работа выполнена при подлержке программой Президента Российской Федерации поддержки ведущих научных школ, грант НШ-8564.2006.10.
\end{abstract}

В параграфе 3 статьи [1] описаны условия сходимости и предельные распределения для произведений случайных величин с значениями в конечной группе, распределения которых определяются конечной простой однородной неразложимой цепью Маркова следующим образом.

Пусть $\mathfrak{I}$ - конечная простая однородная неразложимая цепь Маркова с множеством состояний $\left\{E_{1}, E_{2}, \ldots, E_{n}\right\}$, матрицей переходных вероятностей $P=[p(i, j)]$ и начальным распределением $\left(p_{0}(1), \ldots, p_{0}(n)\right)$. Будем предполагать, что $p_{0}(i)>0$ для всех $i \in\{1, \ldots, n\}$, то есть, что все начальные состояния цепи Маркова $\mathfrak{I}$ возможны.

Пусть $\xi_{i, j}, i, j=1, \ldots, n$, для которых $p(i, j)>0$, суть случайные величины, принимающие значения в некоторой конечной группе $(G, \cdot)$. По случайной реализации $E_{\alpha_{1}}, E_{\alpha_{2}}, \ldots, E_{\alpha_{k+1}}$ цепочки состояний цепи Маркова $\mathfrak{I}$ в первых $k+1$ испытаниях построим произведение

$$
\eta^{(k)}=\varphi_{1} \cdot \varphi_{2} \cdots \varphi_{k},
$$

где случайные величины $\varphi_{1}, \ldots, \varphi_{k}$ независимы и распределение $\varphi_{i}$ совпадает с распределением $\xi_{\alpha_{i}, \alpha_{i+1}}, i=1, \ldots, k$. Для натурального числа $k$ и таких $i, j \in\{1, \ldots, n\}$, что вероятность $p^{(k)}(i, j)$ перехода из $E_{i}$ в $E_{j}$ за $k$ шагов положительна, обозначим через $\eta_{i, j}^{(k)}$ случайную величину, распределение которой совпадает с условным распределением случайной величины $\eta^{(k)}$ при условиях $E_{\alpha_{1}}=E_{i}, E_{\alpha_{k+1}}=E_{j}$.

Будем говорить, что случайные величины $\eta_{i, j}^{(k)}$ есть произведения случайных величин, распределения которых определяются цепью Маркова Э и исходными распределениями случайных величин $\xi_{i, j}, i, j=1, \ldots, n$, для которых $p(i, j)>0$.

$\mathrm{B}$ настоящей работе приведены необходимые и достаточные условия существования такого $k$, что распределения всех определенных при этом $k$ случайных величин $\eta_{i, j}^{(k)}$ со- 
впадают с соответствующими предельными распределениями. В частном случае произведений независимых одинаково распределенных случайных величин эта задача решена Ю. Н. Горчинским в [1].

Введем обозначения, необходимые для точной постановки задачи.

Мы будем использовать понятия теории графов, следуя изложению п. 3.2 главы 2 книги [2]. Введем соответствующий цепи Маркова $\mathfrak{I}$ ориентированный граф $\Gamma$ с множеством вершин $\left\{E_{1}, E_{2}, \ldots, E_{n}\right\}$ и множеством ориентированных ребер $\left\{e_{i, j}^{+1}, e_{i, j}^{-1} \mid i, j \in\{1, \ldots, n\}, p(i, j)>0\right\}$. Так как цепь Маркова $\mathfrak{I}$ неразложима, граф $\Gamma$ сильно связный. Если $\varepsilon=+1$, то $E_{i}-$ начало, а $E_{j}-$ конец $e_{i, j}^{\varepsilon}$, если $\varepsilon=-1$, то $E_{j}$ - начало, а $E_{i}$ - конец $e_{i, j}^{\varepsilon}$.

Путем $z$ в графе $Г$ называется последовательность ребер

$$
z=\left(e_{i_{1}, j_{1}}^{\varepsilon_{1}}, e_{i_{2}, j_{2}}^{\varepsilon_{2}}, \ldots, e_{i_{k}, j_{k}}^{\varepsilon_{k}}\right)
$$

у которой для каждого $s=1,2, \ldots, k-1$ начало ребра $e_{i_{s+1}, j_{s+1}}^{\varepsilon_{s+1}}$ совпадает с концом ребра $e_{i_{s}, j_{s}}^{\varepsilon_{s}}$. При этом начало ребра $e_{i_{1}, j_{1}}^{\varepsilon_{1}}$ есть начало пути $z$, конец ребра $e_{i_{k}, j_{k}}^{\varepsilon_{k}}$ - конец пути $z$.

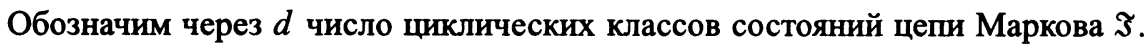

Для $i, j \in\{1, \ldots, n\}$ и целого числа $R$ через $L_{R}(i, j)$ обозначим множество всех путей вида (1) в графе $\Gamma$ с началом $E_{i}$ и концом $E_{j}$, у которых $\varepsilon_{1}+\varepsilon_{2}+\ldots+\varepsilon_{k}=R$. Каждой паре $i, j \in\{1, \ldots, n\}$ однозначно соответствует число $v(i, j) \in\{0, \ldots, d-1\}$, для которого $L_{R}(i, j)$ непусто при $R=v(i, j)+k d$ и любом целом $k$. Зафиксируем произвольно $z_{i, j} \in L_{\nu(i, j)}(i, j), \sigma_{i, j} \in \Omega\left(z_{i, j}\right)$.

Пусть $\gamma(i, j)=(v(1, i)+v(i, j)-v(1, j)) / d$, очевидно, что $\gamma(i, j) \in\{0 ;+1\}$ для всех $i, j \in\{1, \ldots, n\}$.

Пусть

$$
Z^{(1)}=\bigcup_{R=-\infty}^{+\infty} L_{R}(1,1)
$$

- множество петель в $\Gamma$ с концом $E_{1}$.

Для $z \in L_{R}(1,1)$ положим $l(z)=R / d$. Нетрудно видеть, что $l(z)$ - целое число. Для произвольной случайной величины $\xi$ с значениями в группе $G$ обозначим через $\Omega(\xi)=\{g \in G \mid \mathbf{P}(\xi=g)>0\}$.

Далее, для пути $z$ вида (1) построим множество $\Omega(z) \subset G$, полагая

$$
\Omega(z)=\Omega\left(e_{i_{1}, i_{1}}^{\varepsilon_{1}}\right) \ldots \Omega\left(e_{i_{k}, j_{k}}^{\varepsilon_{k}}\right),
$$

где

$$
\Omega\left(e_{s, t}^{\varepsilon}\right)=\Omega\left(\xi_{s, t}\right)^{\varepsilon}
$$

и для подмнсжкеств $M, N \subset G$

$$
M \cdot N=\{\sigma \tau \mid \sigma \in M, \tau \in N\}, \quad M^{-1}=\left\{\sigma^{-1} \mid \sigma \in M\right\} .
$$

Пусть

$$
G_{1}=\bigcup_{z \in Z^{(1)}} \Omega(z),
$$

тогда $G_{1}$ - подгруппа конечной группы $G$. Зафиксируем произвольное $z^{\prime} \in L_{d}(1,1)$, $x_{1} \in \Omega\left(z^{\prime}\right)$ и обозначим через $H_{1}$ минимальную подгруппу среди подгрупп $L$ группы $G$, 
удовлетворяющих соотношению

$$
\Omega(z) \subset x_{1}^{l(z)} \cdot L \quad \forall z \in Z^{(1)} .
$$

Соотношение (2) выполнено при $L=G_{1}$, поэтому $H_{1}$ есть подгруппа $G_{1}$, пусть $r_{1}-$ индекс $H_{1}$ в $G_{1}$.

Согласно основной теореме параграфа 3 в [1], для любого $l \in\left\{0,1, \ldots, r_{1}-1\right\}$ последовательность распределений случайных величин $\eta_{i, j}^{\left(s_{N}\right)}, s_{N}=v(i, j)+\left(l+r_{1} N\right) d$, при $N \rightarrow \infty$ сходится к равновероятному распределению на множестве

$$
\sigma_{1, i}^{-1} \cdot x_{1}^{l+\gamma(i, j)} \cdot H_{1} \cdot \sigma_{1, j}
$$

Нас будут интересовать необходимые и достаточные условия существования такого натурального числа

$$
s=v+d\left(l+N r_{1}\right), \quad v \in\{0, \ldots, d-1\}, \quad l \in\left\{0, \ldots, r_{1}-1\right\}, \quad N \geqslant 0,
$$

что для любых $i, j \in\{1, \ldots, n\}$, удовлетворяющих условию $p^{(s)}(i, j)>0$, распределение случайной величины $\eta_{i, j}^{(s)}$ совпадает с равновероятным распределением на множестве (3). В этом случае мы будем говорить, что имеет место достижимость предельных распределений $\eta_{i, j}^{(k)}$ на конечном шаге $s$.

При доказательстве основной теоремы параграфа 3 в [1] было показано, что для любых $i, j$ и $s$ вида (4), удовлетворяющих условию $p^{(s)}(i, j)>0$, множество

$$
\Omega\left(\eta_{i, j}^{(s)}\right) \subset \sigma_{1, i}^{-1} \cdot x_{1}^{l+\gamma(i, j)} \cdot H_{1} \cdot \sigma_{1, j}
$$

Отсюда следует, что в случае, когда имеет место достижимость предельных распределений на некотором шаге $s$, аналогичное свойство выполняется на шаге $(s+k)$ для любого $k \geqslant 1$. Таким образом, в случае достижимости на конечном шаге, однозначно определено минимальное значение $s$, для которого достижимость выполняется на шаге $s$, такое $s$ будем называть индексом достижимости.

Пусть $K^{*}$ - произвольный класс сопряженных элементов группы $G$, если множество $K=K^{*} \cap G_{1}$ непусто, то $K$ - класс сопряженных элементов группы $G_{1}$. Ввиду соотношения (2), выполненного при $L=H_{1}$, коммутант группы $G_{1}$ содержится в $H_{1}$. Следовательно, $H_{1}$ - нормальный делитель в $G_{1}$, и в силу (2) (при $L=H_{1}$ ), факторгруппа $G_{1} / H_{1}$ циклическая, порожденная смежным классом $x_{1} \cdot H_{1}$. Так как группа $G_{1} / H_{1}$ абелева, для любого натурального $s$ либо $K \subset\left(x_{1}^{s} \cdot H_{1}\right)$, либо $K \cap\left(x_{1}^{s} \cdot H_{1}\right)$ - пустое множество.

Будем обозначать через $f$ максимальную степень неприводимого представления группы $G$ над полем комплексных чисел. Для квадратной матрицы $Q=\left[q_{i, j}\right], i, j \in\{1, \ldots, m\}$, обозначим $\operatorname{Sp}(Q)$ след $q_{1,1}+q_{2,2}+\ldots+q_{m, m}$ матрицы $Q$.

Теорема 1. Достижимость предельных распределений $\eta_{i, j}^{(k)}$ на некотором конечном шаге имеет место тогда и только тогда, когда для всех $s \in\{1, \ldots, n f\}$ таких, что $d$ делит $s$, и любого класса $K^{*}$ сопряженных элементов группы $G$ верно равенство

$$
\sum_{j=1}^{n} p^{(s)}(j, j) \mathbf{P}\left(\eta_{j, j}^{(s)} \in K^{*}\right)= \begin{cases}\operatorname{Sp}\left(P^{s}\right)\left|K^{*} \cap G_{1}\right| /\left|H_{1}\right|, & \text { если } K^{*} \cap G_{1} \subset x_{1}^{s} \cdot H_{1}, \\ 0 & \text { в противном случае. }\end{cases}
$$


Доказательство. Проведем предварительные рассуждения. Пусть

$$
\bar{\xi}_{i, j}=\sigma_{1, i} \cdot \xi_{i, j} \cdot \sigma_{1, j}^{-1}, \quad \forall i, j=1, \ldots, n, \quad p(i, j)>0,
$$

и пусть $\bar{\eta}_{i, j}^{(k)}$ суть произведения случайных величин, определяемых цепью Маркова $\mathfrak{I}$ исходными распределениями $\bar{\xi}_{i, j}, i, j=1, \ldots, n, p(i, j)>0$. Тогда

$$
\bar{\eta}_{i, j}^{(k)}=\sigma_{1, i} \cdot \eta_{i, j}^{(k)} \cdot \sigma_{1, j}^{-1}, \quad \forall k, \quad \forall i, j=1, \ldots, n, \quad p^{(k)}(i, j)>0 .
$$

Из равенств (7) следует, что достижимость предельных распределений $\eta_{i, j}^{(k)}$ на конечном шаге имеет место тогда и только тогда, когда выполнено условие достижимости предельных распределений на конечном шаге для случайных величин $\bar{\eta}_{i, j}^{(k)}$.

Из соотношения (5) следует, что случайные величины $\bar{\xi}_{i, j}, \bar{\eta}_{i, j}^{(k)}$ можно рассматривать как случайные величины со значениями в группе $G_{1}$. Найдем выражение для матричной характеристической функции $\Phi_{\bar{\eta}_{i, j}^{(k)}}(U)$, где $U$ - произвольное неприводимое представление группы $G_{1}$ над полем комплексных чисел (см. §1 в [1]). Пусть $n_{U}-$ степень $U$ и

$$
\Phi(U)=\left[\begin{array}{lll}
\Phi_{1,1}(U) & \ldots & \Phi_{1, n}(U) \\
& \ldots & \\
\Phi_{n, 1}(U) & \ldots & \Phi_{n, n}(U)
\end{array}\right]
$$

- блочная $n n_{U} \times n n_{U}$ матрица, у которой на месте с номером $(i, j)$ стоит нулевая квадратная матрица размера $n_{U}$, если $p(i, j)=0$, и матрица $p(i, j) \Phi_{\bar{\xi}_{i, j}}(U)$, если $p(i, j)>0$. Матрицу $\Phi(U)^{k}, k=1,2, \ldots$, также можно рассматривать как блочную матрищу с $n^{2}$ квадратными блоками $\Phi_{i, j}^{(k)}(U)$ размера $n_{U}$ :

$$
\Phi(U)^{k}=\left[\begin{array}{lll}
\Phi_{1,1}^{(k)}(U) & \ldots & \Phi_{1, n}^{(k)}(U) \\
& \ldots & \\
\Phi_{n, 1}^{(k)}(U) & \ldots & \Phi_{n, n}^{(k)}(U)
\end{array}\right]
$$

Как показано в параграфе 3 в [1], верно равенство

$$
\Phi_{\tilde{\eta}_{i, j}^{(k)}}(U)=\frac{1}{p^{(k)}(i, j)} \Phi_{i, j}^{(k)}(U), \quad p^{(k)}(i, j)>0 .
$$

Вычислим след матриц $\Phi(U)^{d k}, k \geqslant 1$. Из соотношения (8) следует, что для всех $k \geqslant 1$ и всех $U$

$$
\operatorname{Sp}\left(\Phi(U)^{d k}\right)=\sum_{j=1}^{n} p^{(k d)}(j, j) \operatorname{Sp} \Phi_{\bar{\eta}_{j, j}^{(k \cdot d)}}(U),
$$

В правой части (9) в случае $p^{(k d)}(j, j)=0$ соответствующее слагаемое считается равным 0.

По теореме о выражении матричных характеристических функций случайных величин через распределение (см. [1]) и в силу линейности функции след для всех $j \in\{1, \ldots, n\}$, всех $k \geqslant 1$ таких, что $p^{(k d)}(j, j)>0$, и всех $U$ справедливо равенство

$$
\operatorname{Sp}\left(\Phi_{\bar{\eta}_{j, j}^{(k d)}}(U)\right)=\sum_{\sigma \in G} \mathbf{P}\left(\bar{\eta}_{j, j}^{(k d)}=\sigma\right) \operatorname{Sp} U(\sigma) .
$$


Пусть $\chi U$ - неприводимый характер группы $G$, соответствующий представлению $U$, тогда $\operatorname{Sp}(U(\sigma))=\chi U(\sigma)$ для всех $\sigma \in G$, и из (9) и (10) следуют равенства

$$
\operatorname{Sp}\left(\Phi(U)^{d k}\right)=\sum_{\sigma \in G} \chi_{U}(\sigma)\left(\sum_{j=1}^{n} p^{(k d)}(j, j) \mathbf{P}\left(\bar{\eta}_{j, j}^{(k d)}=\sigma\right)\right),
$$

справедливые для всех $k \geqslant 1$ и всех $U$.

Пусть $B$ - множество классов сопряженных элементов группы $G_{1}$. Каждый характер группы постоянен на любом классе сопряженных элементов, поэтому

$$
\chi_{U}(\sigma)=\chi_{U}(K), \quad \forall \sigma \in K, \quad \forall K \in B .
$$

Из соотношений (11) и (12) следует, что для всех $k \geqslant 1$ и всех $U$

$$
\operatorname{Sp}\left(\Phi(U)^{d k}\right)=\sum_{K \in B} \chi_{U}(K)\left(\sum_{j=1}^{n} p^{(k d)}(j, j) \mathbf{P}\left(\bar{\eta}_{j, j}^{(k d)} \in K\right)\right) .
$$

Докажем необходимость условий (6) теоремы. Пусть достижимость предельных распределений $\bar{\eta}_{i, j}^{(k)}$ на конечном шаге $s, s=v+d\left(l+N r_{1}\right)$, имеет место для некоторых $\nu \in\{0,1, \ldots, d-1\}, l \in\left\{0,1, \ldots, r_{1}-1\right\}$ и натурального числа $N$. Тогда для любых $i, j \in\{1,2, \ldots, n\}$ таких, что $p^{(s)}(i, j)>0$, распределение случайной величины $\bar{\eta}_{i, j}^{(s)}$ совпадает с равновероятным распределением на множестве $x_{1}^{l+\gamma(i, j)} \cdot H_{1}$ (см. (3) и (7)).

Рассмотрим случай, когда ядро $\operatorname{Ker} U$ неприводимого представления $U$ группы $G_{1}$ не содержит $H_{1}$. Обозначим через $\eta$ случайную величину со значениями в $G_{1}$, имеющую равновероятное распределение на множестве $H_{1}$. Разлагая ограничение $\operatorname{Res}_{H_{1}} U$ представления $U$ на подгруппу $H_{1}$ на неприводимые компоненты и используя теорему о выражении характеристических функций через распределение (см. §1 в [1]) и теорему Клиффорда [3], получаем соотношение

$$
\Phi_{\eta}(U)=O_{n_{U} \times n_{U}} \quad \forall U: \operatorname{Ker} U \not \supset H_{1}
$$

В правой части соотношения (14) стоит нулевая квадратная матрица размера $n_{U}$. Следовательно, для всех $i, j \in\{1,2, \ldots, n\}$ таких, что $p^{(s)}(i, j)>0$,

$$
\Phi_{\bar{\eta}_{i, j}^{(s)}}(U)=U\left(x_{1}^{l+\gamma(i, j)}\right) \cdot \Phi_{\eta}(U)=O_{n_{U} \times n_{U}} .
$$

Из соотношений (8), (15) следует, что для всех $U$ таких, что $\operatorname{Ker} U \not \supset H_{1}$,

$$
\Phi(U)^{s}=O_{n} n_{U} \times n^{n}
$$

Из условия (16) вытекает, что все собственные числа матрицы $\Phi(U)$ равны 0 , но тогда то же верно и для всех собственных чисел матриц $\Phi(U)^{k}, k \geqslant 1$. Таким образом, для всех $k \geqslant 1$ и всех $U$ таких, что $\operatorname{Ker} U \not \supset H_{1}$,

$$
\operatorname{Sp}\left(\Phi(U)^{d k}\right)=0
$$

Из соотношений (13) и (17) следует, что для всех $k \geqslant 1$ и всех $U$ таких, что $\operatorname{Ker} U \not \supset H_{1}$,

$$
\sum_{K \in B} \chi_{U}(K)\left(\sum_{j=1}^{n} p^{(k d)}(j, j) \mathbf{P}\left(\bar{\eta}_{j, j}^{(k d)} \in K\right)\right)=0
$$


Пусть теперь $\operatorname{Ker} U \supset H_{1}$. Так как $G_{1} / H_{1}$ - циклическая группа, порожденная смежным классом $x_{1} \cdot H_{1}$ порядка $r_{1}$, ясно, что $U$ - одномерное представление, $U=\chi U$, $\chi U\left(x_{1}\right)^{r_{1}}=1$. Из соотношений (5) и (7) с учетом равенства $\gamma(j, j)=0$, справедливого для всех $j=1, \ldots, n$, следует, что для всех $j=1, \ldots, n$ и $k \geqslant 1$ таких, что $p^{(k d)}(j, j)>0$, и всех $U$ таких, что $\operatorname{Ker} U \supset H_{1}$, справедливо равенство

$$
\Phi_{\bar{\eta}_{j, j}^{(k d)}}(U)=\chi_{U}\left(x_{1}^{k}\right)
$$

Согласно (8) и (19)

$$
\operatorname{Sp}\left(\Phi(U)^{k d}\right)=\operatorname{Sp}\left(P^{k d}\right) \cdot \chi_{U}\left(x_{1}^{k}\right)
$$

для всех $k \geqslant 1$ и всех $U$ таких, что $\operatorname{Ker} U \supset H_{1}$.

Из соотношений (13) и (20) получаем, что для всех $k \geqslant 1$ и всех $U$ таких, что $\operatorname{Ker} U \supset H_{1}$, справедливо равенство

$$
\sum_{K \in B} \chi U(K)\left(\sum_{j=1}^{n} p^{(k d)}(j, j) \mathbf{P}\left(\bar{\eta}_{j, j}^{(k d)} \in K\right)\right)=\operatorname{Sp}\left(P^{k d}\right) \cdot \chi_{U}\left(x_{1}^{k}\right) .
$$

Зафиксируем $k \geqslant 1$ и рассмотрим систему линейных уравнений относительно неизвестных $y_{K}, K \in B$,

$$
\sum_{K \in B} \chi_{U}(K) \cdot y_{K}= \begin{cases}0, & \operatorname{Ker} U \not \supset H_{1}, \\ \operatorname{Sp}\left(P^{k d}\right) \cdot \chi_{U}\left(x_{1}^{k}\right), & \operatorname{Ker} U \supset H_{1} .\end{cases}
$$

Так как число неприводимых характеров конечной группы совпадает с числом ее классов сопряженных элементов, число уравнений в системе (22) совпадает с числом неизвестных. Ввиду линейной независимости характеров, матрица системы (22) является невырожденной, и данная система имеет единственное решение, которым, в силу (18) и (21), является набор значений

$$
y_{K}=\sum_{j=1}^{n} p^{(k d)}(j, j) \mathbf{P}\left(\bar{\eta}_{j, j}^{(k d)} \in K\right), \quad K \in B .
$$

Используя теорему Клиффорда, можно показать, что решением (22) также является набор значений $y_{K}, K \in B$, где

$$
y_{K}= \begin{cases}\left(|K| /\left|H_{1}\right|\right) \cdot \operatorname{Sp}\left(P^{k d}\right), & K \subset x_{1}^{k} \cdot H_{1} \\ 0, & K \not \subset x_{1}^{k} \cdot H_{1}\end{cases}
$$

Система уравнений (22) имеет единственное решение, поэтому для всех $K \in B$ и $k \geqslant 1$

$$
\sum_{j=1}^{n} p^{(k d)}(j, j) \mathbf{P}\left(\bar{\eta}_{j, j}^{(k d)} \in K\right)= \begin{cases}\left(|K| /\left|H_{1}\right|\right) \cdot \operatorname{Sp}\left(P^{k d}\right), & K \subset x_{1}^{k} \cdot H_{1}, \\ 0, & K \not \subset x_{1}^{k} \cdot H_{1} .\end{cases}
$$

Для каждого класса $K$ сопряженных элементов подгруппы $G_{1}$ однозначно определен класс $K^{*}$ сопряженных элементов группы $G$ такой, что $K=K^{*} \cap G_{1}$, при этом, согласно соотношениям (5), (7), при $i=j$ для всех $j \in\{1, \ldots, n\}$ и для всех $k \geqslant 1$ таких, что 
$p^{(k d)}(j, j) \geqslant 0$, события $\left\{\bar{\eta}_{j, j}^{(k d)} \in K\right\}$ и $\left\{\eta_{j, j}^{(k d)} \in K^{*}\right\}$ совпадают и их вероятности равны между собой.

Таким образом, справедливость условия (6) следует из доказанного соотношения (24).

Докажем достаточность условий (6) для достижимости предельных распределений $\eta_{i, j}^{(k)}$ на конечном шаге.

Из условий (6) следуют равенства (24), а тогда набор значений (23) есть решение системы (22) для любого натурального числа $k$ такого, что $s=k d \leqslant n f$. В частности, при этих же значениях $k$ выполняются равенства (18), а с ними (с учетом (13)) и равенства (17).

Заметим, что $\operatorname{Sp}\left(\Phi(U)^{s}\right)=0$, если $d$ не делит $s$, таким образом, для всех $s \in$ $\{1, \ldots, n f\}$ и всех $U$ таких, что $\operatorname{Ker} U \not \supset H_{1}$, справедливо равенство

$$
\operatorname{Sp}\left(\Phi(U)^{s}\right)=0
$$

Рассмотрим характеристический многочлен $f_{U}(\lambda)$ матрицы $\Phi(U)$, его коэффициенты с помощью формул Ньютона можно выразить в виде значений многочленов (с нулевыми свободными членами) от чисел $\operatorname{Sp}\left(\Phi(U)^{s}\right), s=1,2, \ldots, n n_{U}$. Но тогда, поскольку $n_{U} \leqslant f$, из (25) следует, что $f_{U}(\lambda)=\lambda^{n_{U}}$, и по теореме Гамильтона-Кэли

$$
\Phi(U)^{n n_{U}}=O_{n n_{U} \times n n_{U}}
$$

для всех $U$ таких, что $\operatorname{Ker} U \not \supset H_{1}$.

Из соотношений (26) и (8) получаем, что для всех $i, j=1, \ldots, n$ таких, что $p^{(n f)}(i, j)>0$, и для всех $U$ таких, что $\operatorname{Ker} U \not \supset H_{1}$, справедливо равенство

$$
\Phi_{\bar{\eta}_{i, j}^{(n f)}}(U)=O_{n_{U} \times n_{U}}
$$

Так как, в силу (5) и (7), множество $\Omega\left(\bar{\eta}_{i, j}^{(n f)}\right)$ содержится в одном смежном классе группы $G_{1}$ по ее подгруппе $H_{1}$, из (27) следует, что распределение $\bar{\eta}_{i, j}^{(n f)}$ совпадает с равновероятным распределением на соответствующем смежном классе $G_{1}$ по $H_{1}$ для всех $i, j=1, \ldots, n$ таких, что $p^{(n f)}(i, j)>0$. Таким образом, для случайных величин $\bar{\eta}_{i, j}^{(k)}$, a с ними и для случайных величин $\eta_{i, j}^{(k)}$ имеет место достижимость предельных распределений на конечном шаге $n f$.

Теорема доказана.

Следствие 1. Если достижимость предельных распределений $\eta_{i, j}^{(k)}$ на конечном шаге имеет место, то индекс достижимости не превосходит $n f$.

Заметим, что, согласно доказательству теоремы 1 , в качестве значения $f$ в теореме 1 и следствии 1 можно рассматривать максимальную степень неприводимого представления подгруппы $G_{1}$ группы $G$.

Будем говорить, что для случайных величин $\eta_{i, j}^{(k)}$ имеет место достижимость равновероятного распределения на группе $G$ на конечном шаге, если существует натуральное число $s$ такое, что для всех $i, j \in\{1, \ldots, n\}$, для которых $p^{(s)}(i, j)>0$, распределение случайной величины $\eta_{i, j}^{(s)}$ совпадает с равновероятным распределением на группе $G$; минимальное $s$ с указанным свойством назовем индексом достижимости на конечном шаге равновероятного распределения на группе $G$. 
Следствие 2. Достижимость равновероятного распределения на группе $G$ на конечном шаге для случайных величин $\eta_{i, j}^{(k)}$ имеет место тогда и только тогда, когда для любого числа $s \in\{1, \ldots, n f\}$ такого, что $d$ делит $s$, и любого класса $K^{*}$ сопряженных элементов группы $G$ верно равенство

$$
\sum_{j=1}^{n} p^{(s)}(j, j) \mathbf{P}\left(\bar{\eta}_{j, j}^{(s)} \in K^{*}\right)=\operatorname{Sp}\left(P^{s}\right) \frac{\left|K^{*}\right|}{|G|}
$$

Если достижимость равновероятного распределения на группе $G$ имеет место, то индекс достижсиости не превосходит $n f$.

Доказательство следствия 2 проводится аналогично доказательству теоремы 1 , при этом всюду в качестве представления $U$ рассматривается некоторое неприводимое комплексное представление группы $G$ и условие $\operatorname{Ker} U \not \supset H_{1}$, заменяется на условие, что $U$ есть неединичное представление.

Для случая положительно-регулярных цепей Маркова утверждение следствия 2 было доказано Ю. Н. Горчинским.

\section{Список литературы}

1. Горчинский Ю. Н., Круглов И. А., Капитонов В. М., Вопросы теории распределений на конечных группах. Труды по дискретной математике (1997) 1, 85-112.

2. Бахтурин Ю. А. Основные структуры современной алгебры. Наука, Москва, 1990.

3. Кэртис Ч., Райнер И., Теория представлений конечных групп и ассочиативных алгебр. Наука, Москва, 1964.

Статья поступила 14.03.2006. 